\title{
Inclusive NC and CC diffraction
}

\section{Laurent Schoeffel* ${ }^{*}$}

CEA Saclay, France

E-mail: schoffel@hep.saclay.cea.fr

Recent diffractive structure function measurements in deep inelastic scattering (DIS) by the H1 and ZEUS experiments at HERA are reviewed. In neutral current (NC) DIS, precise measurements have been made of diffraction, $e p \rightarrow e X Y$, the signature of which is a large rapidity gap (LRG) between the hadronic system $X$ and the proton or one of its low-mass excited states $Y$. New results on charged current (CC) DIS are also presented. They are identified in a CC DIS sample by a large rapidity gap, corresponding to a diffractive process $e p \rightarrow \nu X Y$ with a $W$ boson exchange. Moreover, within the framework of QCD hard scattering factorisation in DIS, all these data provide constraints on the diffractive parton distribution functions of the proton and predict a large gluon content. For example, the resulting gluon distribution, extracted from the $\mathrm{H} 1$ measurements, carries an integrated fraction of about $70 \%$ of the exchanged momentum. Note that these functions are crucial inputs for the calculations of diffractive dijet or charm production in DIS. These parton distribution functions may also be applicable to hadron-hadron scattering provided that an additional rapidity gap survival probability is taken into account.

International Europhysics Conference on High Energy Physics

July 21st - 27th 2005

Lisboa, Portugal

\footnotetext{
${ }^{*}$ Speaker.

${ }^{\dagger}$ On behalf of the $\mathrm{H} 1$ and ZEUS collaborations
} 


\section{Measurements in NC DIS and interpretation}

At low $x$ in DIS at HERA, approximately $10 \%$ of the events are of the type $e p \rightarrow e X p$, where the final state proton carries more than $95 \%$ of the proton beam energy. For these processes, a photon of virtuality $Q^{2}$, coupled to the electron (or positron), undergoes a strong interaction with the proton (or one of its low-mass excited states $Y$ ) to form a hadronic final state system $X$ (of mass $M_{X}$ ) separated by a LRG from the leading proton, or the system $Y$ (of mass $M_{Y}$ ). In such a reaction, $e p \rightarrow e X Y$, no net quantum number are exchanged and a fraction $x_{I P}$ of the proton longitudinal momentum is transferred to the system $X$. In addition, the virtual photon couples to a quark carrying a fraction $\beta=\frac{x}{x_{I P}}$ of the exchanged momentum. Extensive measurements of diffractive DIS cross sections have been made by both the ZEUS and H1 collaborations [1, 2, 3, 4], using different techniques : by requiring explicitely the presence of a LRG, by exploiting the specific $M_{X}$ distribution of diffractive events, ot by tagging the $Y$ system in dedicated detectors. The results are presented in Fig. 1.

Events with the diffractive topology can be analysed in Regge models in terms of pomeron trajectory exchange between the proton and the virtual photon. However, the large virtualities $Q^{2}$ encourage a perturbative QCD treatment. Then, the diffractive component of DIS is analysed in terms of parton distribution functions (PDFs). According to the QCD factorisation theorem [5], these diffractive PDFs (see Fig. 2) will also undergo QCD evolution as a function of $Q^{2}$ in the same way as the inclusive proton PDFs.

\section{Measurements in CC DIS}

Events with a LRG have been observed in $e p$ CC DIS with the H1 and ZEUS detectors [6, 7]. Both experiments have presented cross sections differentially in $Q^{2}, x_{I P}$ and $\beta$. The ratio of diffractive to inclusive $\mathrm{NC}$ cross sections has been determined at $Q^{2}>200 \mathrm{GeV}^{2}$ and is compared to that for the CC process measured in the same kinematic region in Fig. 3 for ZEUS results. About 2 to $4 \%$ of the CC events are diffractive, as also measured by the $\mathrm{H} 1$ experiment. Both collaborations have produced a total $\mathrm{CC}$ cross section in the kinematic range of the analysis, giving for $\mathrm{H} 1 \sigma_{C C}^{\text {diff }}=0.42 \pm 0.13($ stat $) \pm 0.09($ syst $) \mathrm{pb}$, in good agreement with the RAPGAP MonteCarlo prediction.

\section{References}

[1] S. Chekanov et al. [ZEUS Collaboration], Nucl. Phys. B713 3 (2005).

[2] S. Chekanov et al. [ZEUS Collaboration], Eur. Phys. J. C38 43 (2004).

[3] H1 Collaboration, contributed paper to ICHEP 2002, Amsterdam, H1 prelim-02-012.

[4] H1 Collaboration, contributed paper to EPS 2001, Budapest, H1 prelim-01-112.

[5] J. Collins, Phys. Rev. D57 3051 (1998). Erratum ibid D61 (2000) 019902.

[6] ZEUS Collaboration, contributed paper to LP 2005, Uppsala, ZEUSprelim-05-004.

[7] H1 Collaboration, contributed paper to ICHEP 2004, Beijing, H1 prelim-04-115. 


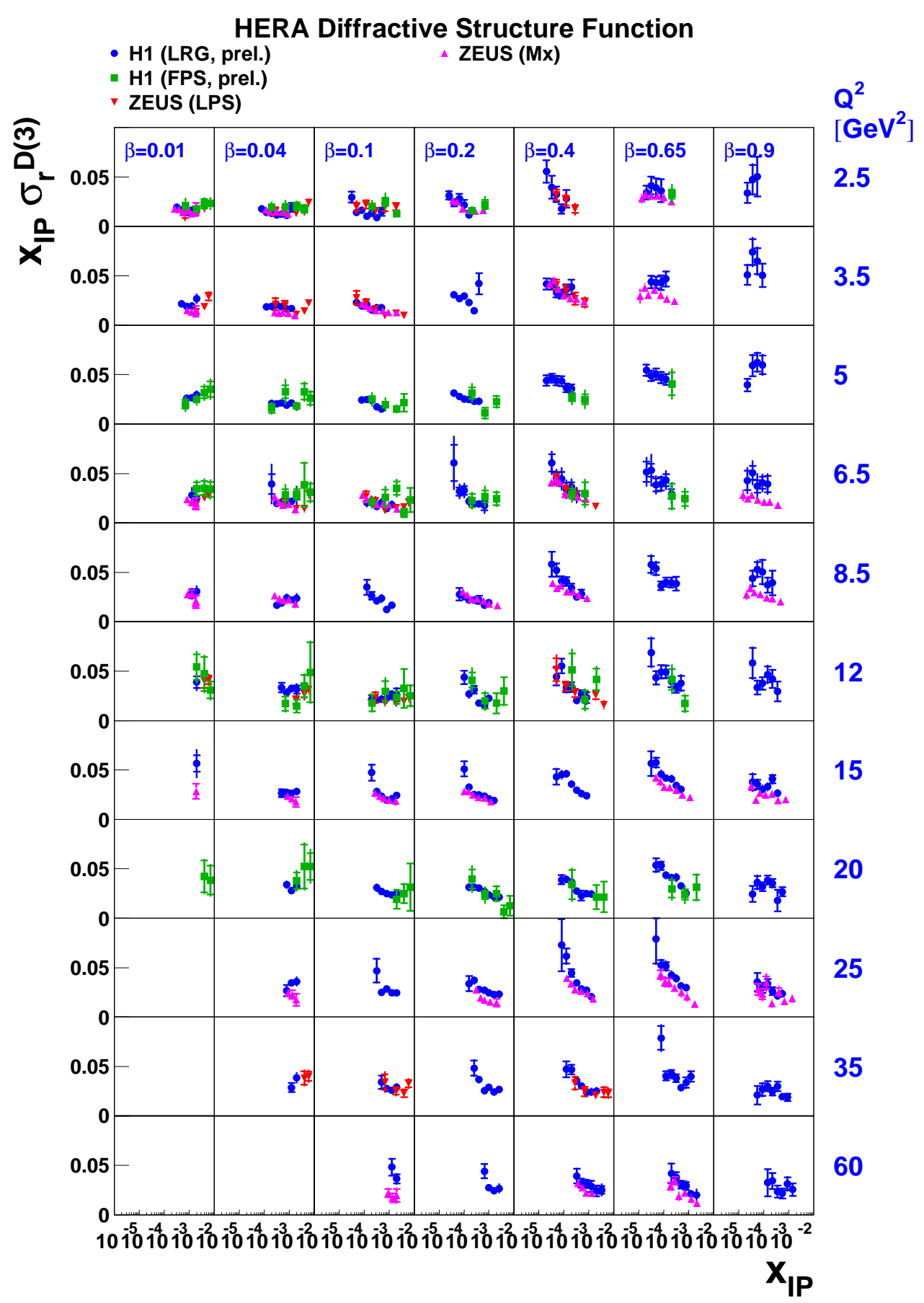

Figure 1: Summary of all diffractive DIS data sets at medium $Q^{2}$, for $x_{I P} \sigma_{r}^{D(3)} \simeq x_{I P} F_{2}^{D(3)}$ in $Q^{2}, \beta$ bins as a function of $x_{I P}$, with $\sigma_{r}^{D(3)}=\frac{x Q^{4}}{4 \pi \alpha_{e m}^{2}} \frac{1}{\left(1-y+\frac{y^{2}}{2}\right)} \frac{\mathrm{d}^{3} \sigma_{\epsilon p \rightarrow e X Y}}{\mathrm{~d} x_{I P} \mathrm{~d} x \mathrm{~d} Q^{2}}$. The $Q^{2}$ and $\beta$ values have been shifted to the $\mathrm{H} 1$ bin centers using small translation factors. Note that all sets have been converted to the measurement range $M_{Y}<1.6 \mathrm{GeV}$. Thus, ZEUS- $M_{X}$ data, published for $M_{Y}<2.3 \mathrm{GeV}$, have been multiplied by a universal factor 0.77 . The LPS and FPS data (leading proton measurements) have been multiplied by factor of 1.1 , which takes into account the conversion from elastic scattering to $M_{Y}<1.6$ $\mathrm{GeV}$. Relative normalisation uncertainties of order $10 \%$ due to these factors are not shown. 


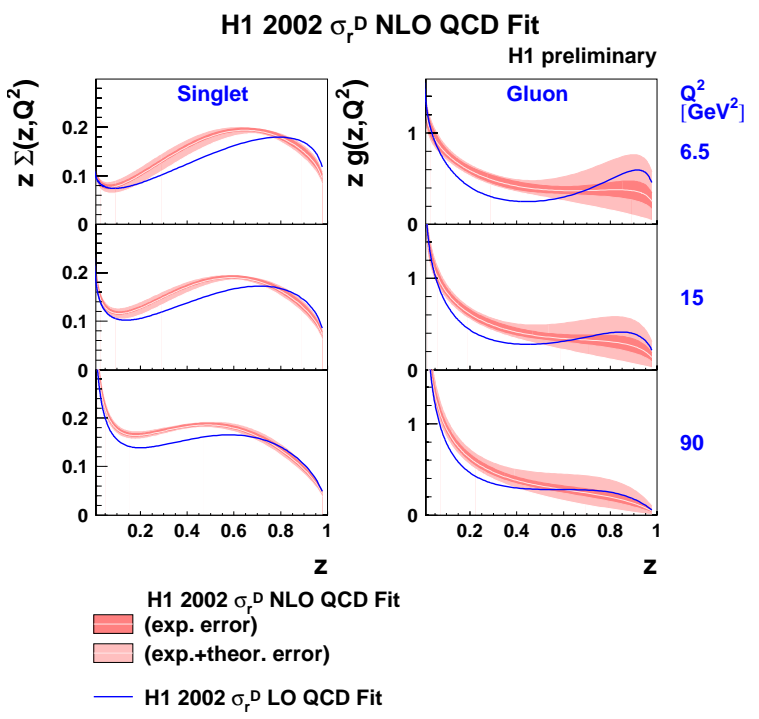

Figure 2: Diffractive parton densities obtained from the QCD fit of the H1 data, normalised such that the pomeron flux is unity at $x_{I P}=0.003$. The left hand side shows the singlet quark distribution (6u where we assume $u=d=s=\bar{u}=\bar{d}=\bar{s}$ ). The right hand side shows the gluon density. The red bands show the results of the NLO fits, with inner error bands showing the experimental errors (statistical and systematic) and the other error bands showing the full uncertainties, including those arising from the theoretical assumptions.

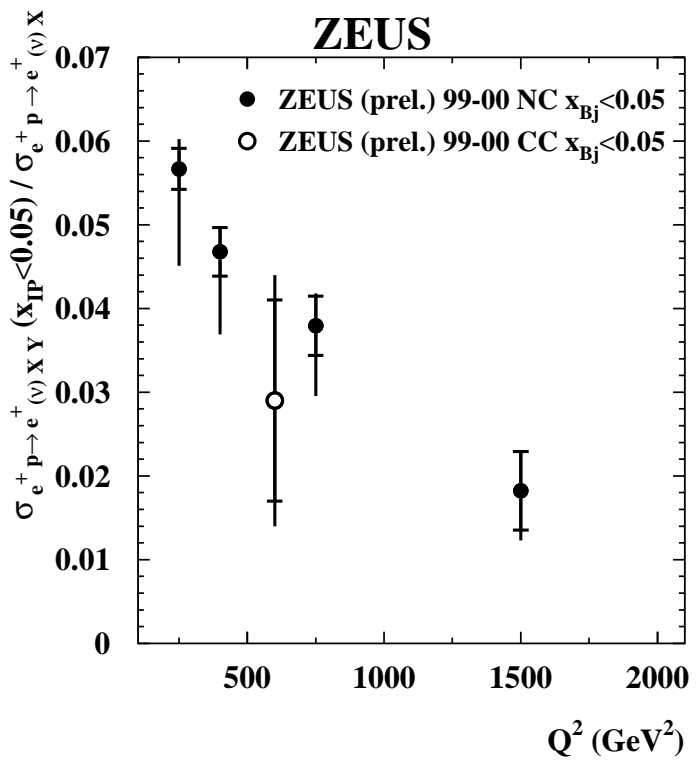

Figure 3: The ratio of the NC LRG cross section $\sigma^{L R G}(e p \rightarrow e X Y)$ to the total cross section $\sigma^{\text {tot }}(e p \rightarrow e X)$ as a function of $Q^{2}$ is compared to the corresponding ratio for CC. Similar results are also found by the H1 experiment. 